\title{
La gestión de recursos humanos en contextos de individualización ${ }^{*}$
}

\author{
Human Resources Management \\ in Contexts of Individualization
}

\author{
Juan Javier Vesga Rodríguez \\ ORCID: 0000-0003-2382-5104 \\ Universidad Católica de Colombia
}

Recibido: 17 de febrero de 2019 Revisado: 6 de abril de 2019 Aceptado: 4 de junio de 2019

\section{Resumen}

La sociedad actual y el mundo del trabajo han sufrido procesos de transformación caracterizados por una tendencia hacia la individualización en las relaciones y la fragilización de los vínculos humanos. Este contexto demanda para las organizaciones retos importantes para la gestión de recursos humanos, debido a que se deben replantear las estrategias y políticas dirigidas a la gestión de personas basada en los colectivos, para remplazarlas por prácticas de gestión individualizadas que respondan a las características actuales de las relaciones de trabajo. En este artículo se proponen un conjunto de reflexiones sobre las implicaciones en la gestión de recursos humanos derivadas de los procesos de individualización y se sugieren algunas alternativas como los contratos idiosincrásicos, el liderazgo individualizado y la gestión de los contratos psicológicos como formas más efectivas de gestionar los recursos humanos en las organizaciones en contextos de individualización.

Palabras clave: gestión de recursos humanos; individualización; relaciones de Trabajo.

Artículo de investigación. Citar como: Vesga, R. J. J. (2020). La gestión de Recursos Humanos en contextos de individualización. Diversitas: Perspectivas en Psicología, 16(1), 201-213. Dol: https://doi.org/10.15332/22563067.5551

Autor de correspondencia: Juan Javier Vesga, Universidad Católica de Colombia. Director Doctorado en Psicología. Dirección postal: Avenida Caracas 46-40 Bogotá D.C., Colombia. Correo electrónico: jjvesga@ucatolica.edu.co; juanjaviervesga@gmail.com 


\section{Abstract}

Current society and the world of labor have undergone processes of transformation characterized by a tendency towards individualization in relationships and the weakening of human bonds. This context poses important challenges to organizations' human resources management, in the sense that strategies and policies aimed at the management of people based on collectives must be rethought, so as to replace them with individualized management practices that respond to the characteristics of current work relations. This article proposes a set of reflections on the implications for the management of human resources derived from the processes of individualization and suggests some alternatives such as idiosyncratic contracts, individualized leadership and the management of psychological contracts as more effective ways of managing human resources in organizations in individualization contexts.

Keywords: human resources management; individualization; work relationships.

\section{Introducción}

Una de las características que tipifica las sociedades industrializadas actuales es la transformación que han tenido en la construcción de vínculos y formas de relacionamiento tanto entre individuos como entre estos y las colectividades a las que pertenecen. Se evidencia una creciente tendencia hacia el debilitamiento y fragilidad de los vínculos humanos y la individualización en las relaciones e interacciones sociales, según se desprende de las reflexiones y discusiones propuestas por diversos autores, especialmente desde la Sociología y la Psicología (Bauman, 2007; Beck, 1998; Beck \& Beck-Gernsheim, 2003; Castells, 1999; Malvezzi, 2011; Soto, 2009).

La fragilidad de estos vínculos ha sido puesta en escena para la discusión por autores como Bauman (2007), para quien "los vínculos durables despiertan su sospecha de una dependencia paralizante” ( $p$. 70). Este autor describe la creciente fragilidad de los vínculos interpersonales debida a una concepción de estos como lazos que atan y dificultan el desarrollo de la propia individualidad, lo que lleva a "establecer 'relaciones de bolsillo', que 'se pueden sacar en caso de necesidad', pero que también pueden volver a sepultarse en las profundidades del bolsillo cuando ya no son necesarias" (p. 10). En esta misma línea, en reemplazo de las formas tra- dicionales de relacionamiento cara a cara, emergen de manera acelerada las relaciones virtuales que "son de fácil acceso y salida. Parecen sensatas e higiénicas, fáciles de usar y amistosas con el usuario, cuando se las compara con la 'cosa real', pesada, lenta, inerte y complicada” (Bauman, 2007, p. 13).

En cuando a la individualización, si bien este fenómeno se presenta con mayor fuerza en los años de transición del siglo $x x$ al xxı, según lo manifiesta Sánchez (2008) este asunto ya "ha sido abordado por autores como Max Weber (1903), Georges Simmel (1908) y Norbert Elias (1987), entre otros" (p. 210). Uno de los autores contemporáneos más reconocidos en el tema de la individualización es Ulrich Beck. Como lo afirma este autor, en la individualización "el propio individuo se convierte en la unidad de reproducción vital de lo social [...] de modo que los individuos [...] se convierten en los sujetos de su subsistencia, mediada por el mercado, y de su organización y planificación biográficas" [cursivas en el original] (Beck, 1988, p. 166).

Paralelo a estos procesos macrosociales, existen otras dinámicas económicas, tecnológicas y políticas que han impactado la manera como se estructuran y gestionan las organizaciones y las relaciones alrededor del trabajo. Específicamente, en la gestión de los recursos humanos se evidencia el crecimiento de la flexibilidad laboral (Gómez, 2014), la desin- 
dicalización (Boltanski \& Chiapello, 2002), la fragilización de la acción colectiva (Rodríguez, Erbes, Roitter, \& Pujol, 2015), la disminución de los vínculos trabajadores-organización (Malvezzi, 2011), el debilitamiento de las identidades colectivas laborales (Vesga, 2019) y la emergencia del concepto de empleabilidad (Rentería, 2012), entre otros. Estas condiciones producen nuevas formas individualizadas de relacionamiento entre los trabajadores y las organizaciones, lo que pone en duda la efectividad de las estrategias tradicionales colectivistas de gestión de los recursos humanos. Como lo ha afirmado Pfeffer (2000) los "cambios en la forma y el funcionamiento organizacional ponen en entredicho las teorías de la organización que se han desarrollado para explicar un contexto que ha variado" (p. 22).

Las organizaciones requieren del involucramiento y compromiso de los trabajadores con sus organizaciones, y que los departamentos de Recursos Humanos desarrollen estrategias y políticas orientadas a este propósito. Sin embargo, los líderes organizacionales encuentran dificultades al querer lograr el compromiso de todos los trabajadores por igual, puesto que las estrategias de gestión están diseñadas para impactar colectivos, lo que se contradice con las características de individualización en las relaciones de trabajo que predominan en la actualidad. Este artículo se orienta a discutir la necesidad de replantear las estrategias de gestión de recursos humanos al interior de las organizaciones, para tener una mayor coherencia entre estas y las características actuales del mundo del trabajo y así lograr mayor efectividad en el alcance de los objetivos organizacionales a través de la gestión de los trabajadores.

\section{Procesos de individualización}

Como se mencionó en la introducción, varios autores señalan que el proceso de individualización se ha venido dando en las sociedades industrializadas, especialmente en el paso de la modernidad a la posmodernidad. El desarrollo de la individualización se vincula con el proceso histórico de diferenciación social propio de esta transición y se expresa en un conjunto de creencias, prácticas culturales y normas sociales características de las sociedades industrializadas (Zabludovsky, 2013). Como lo men- ciona esta autora, "el problema del individualismo y el de la individualización han sido recuperados de forma creciente para hacer un diagnóstico de lo que ocurre en la sociedad contemporánea" (p. 245).

Una de las bases para la comprensión de los procesos de individualización en las sociedades actuales puede encontrarse en Durkheim (2012) en su obra La división del trabajo social'. Durkheim hace una distinción entre la solidaridad mecánica, propia de las sociedades premodernas, y la sociedad orgánica presente en las sociedades modernas. La primera deriva de la semejanza entre los individuos y se fundamenta en una fuerte conciencia colectiva, mientras que la segunda deriva de la complementariedad y se basa en la división del trabajo (Ribes, 2012).

El concepto de solidaridad orgánica es fundamental en el entendimiento de los procesos de individualización en la perspectiva de Durkheim. Para este autor, el paso que las sociedades tienen desde la solidaridad mecánica a la orgánica produce un debilitamiento de los lazos sociales que resultan de la semejanza entre los individuos, y "es la división del trabajo la que llena cada vez más la función que antes desempeñaba la conciencia común" (Durkheim, 2012, p. 223), como mecanismo para evitar la disolución de la sociedad. De esta manera, la división del trabajo se convierte en la característica central que tipifica las sociedades modernas, con su correspondiente especialización de las ocupaciones y las industrias que se presentaban ya en aquella época como producto de la segunda revolución industrial, y que para Durkheim ocurre no solo en el ámbito económico sino también en lo político, lo administrativo, lo artístico, pero ante todo en lo científico, pues la ciencia "se ha fragmentado en una multitud de disciplinas especiales, cada una con su objeto, su método, su espíritu” (Durkheim, 2012, p. 106).

La individualización es ese proceso social que transforma masas de seres humanos en grupos de individuos en los cuales cada uno emerge como una unidad diferenciada del resto de la colectividad. Es un proceso de fragmentación de grandes colectivos en donde los individuos son indiferenciados para lo-

1 La obra se publica inicialmente en 1893, pero es solo con la publicación de la segunda edición en 1902 que el texto queda definitivamente fijado como se conoce actualmente (Ribes, 2012). 
grar una mayor diferenciación de estos para lograr una identidad personal. Sin embargo, esta fragmentación no implica la disolución de la sociedad como estructura humana, sino que esta se transforma para mantenerse unificada sin invisibilizar al individuo que la conforma, pues la sociedad se mantiene unida haciendo uso de la complementariedad diferenciada de la totalidad de los individuos. Al hacer más visibles las particularidades individuales se fortalecen las dinámicas de complementariedad que son la base para una integración basada en las diferencias.

La individualización como proceso social no debe ser confundido con la individuación, que implica la formación de un individuo como persona, como ser único (Beck, 1998). La individuación, término que proviene de la teoría psicológica de Carl de Jung, es un proceso psicológico por el cual un ser humano llega a ser un individuo, es decir, una unidad única, indivisible, separada, un todo (Jung, 1946). De otra parte, la individualización es una categoría socio-histórica e histórico-sociológica, referida no a la conciencia o a la identidad, sino a procesos y transformaciones sociales cuyo foco de atención e incidencia no son el colectivo sino el individuo (Beck, 1998). En este sentido, las instituciones y estructuras de poder orientan sus estrategias y políticas para impactar con sus acciones más que a las colectividades, a los individuos.

“Individualización” significa, en primer lugar, el proceso de desvinculación [disembedding] y, en segundo lugar, el proceso de revinculación a nuevas formas de vida de la sociedad industrial en sustitución de las antiguas, en las que los individuos deben producir, representar y combinar por sí mismos sus propias biografías. De ahí el nombre “individualización”. La desvinculación y revinculación (por utilizar los términos de Giddens) no ocurren por casualidad, ni voluntariamente, ni a través de diversos tipos de condiciones históricas, sino de una vez y en las condiciones generales del estado de bienestar en la sociedad industrial avanzada, tal como ha evolucionado a partir de los años sesenta en numerosos países occidentales (Beck, 1997, pág. 28).

La individualización, por tanto, no es una elección libre y soberana de los individuos, sino que tal con- dición es impuesta por las dinámicas del mercado y las instituciones dominantes. De esta manera, se habla de "individualismo institucionalizado" (Beck \& Beck-Gernsheim, 2003): "Las instituciones cardinales de la sociedad moderna -los derechos civiles, políticos y sociales básicos, pero también el empleo remunerado y la formación y movilidad que éste conlleva- están orientadas al individuo y no al grupo" (pág. 30). En este orden de ideas, el Estado y las instituciones dominantes promulgan las normas, políticas y directrices que gobiernan las actuaciones de los individuos en el desarrollo y construcción de su propia biografía. Así, la individualización institucionalizada implica que la construcción de las propias biografías está matizada por las condiciones y directrices impuestas por las instituciones dominantes.

Un claro ejemplo de los procesos de individualización en la economía actual son las estrategias de mercadeo dirigidas de manera personalizada a cada individuo. Esto se logra a partir de los análisis de los comportamientos y las pautas de consumo registradas de los individuos a través de las tecnologías de comunicaciones y toda la información obtenida a través de su navegación en la red, por las consultas que hace en sitios web, las aplicaciones que descarga y usa y, en general, la información que entrega por su permanente conectividad en la red.

Igualmente, en distintos escenarios académicos, tanto de educación formal como no formal, abundan acciones de capacitación y publicaciones de libros dirigidas a convencer a los individuos de crear su propia empresa, ser emprendedores, desarrollar su proyecto de vida, con el trasfondo de una filosofía popular de que cada uno es el "artífice de su propio destino".

Como lo afirman Beck y Beck-Gernsheim (2003), la individualización lleva impresa la filosofía del "hágalo usted mismo", una necesidad compulsiva de vivir la propia vida, tener el dinero propio y su espacio propio. En consecuencia, el éxito o el fracaso son un resultado completamente individual, y de esta manera, "los problemas sociales pueden convertirse directamente en estados anímicos: en sentimientos de culpabilidad, ansiedades, conflictos y neurosis [...]. Las crisis sociales aparecen como algo individual y ya no son percibidas -0 solo de 
manera indirecta- en su dimensión social” (Beck \& Beck-Gernsheim, 2003, p. 73).

\section{Individualización en las relaciones de trabajo}

Las primeras seis décadas del siglo $x x$-con excepción de los años de la Gran depresión-, pero en particular los años de la posguerra a partir de 1945, estuvieron caracterizados por condiciones laborales de seguridad y estabilidad. Fue una época en que se decía que "la seguridad forma parte, por lo tanto, de la definición implícita, pero admitida por todos, del contrato de trabajo" (Boltanski \& Chiapello, 2002, p. 138).

Pero a partir de la década de los años 70, la economía mundial y los sistemas productivos y de gestión experimentaron un proceso de transformación importante acerca de la manera de comprender, estructurar y administrar las organizaciones, pues se puso de manifiesto la incapacidad del fordismo ${ }^{2}$ para mantener la estabilidad de las empresas, evidenciada principalmente por la rigidez en varios sentidos. Primero, en las inversiones de largo plazo, de capital fijo en los sistemas de producción en masa. Segundo, en los mercados de la fuerza de trabajo y en los contratos laborales, entre otros, lo que llevó a un viraje desde el fordismo a la "acumulación flexible" (Harvey, 1998). En este modelo se "apela a la flexibilidad con relación a los procesos laborales, los mercados de la mano de obra, los productos y las pautas de consumo" (Harvey, 1998, p. 170).

La flexibilidad se convirtió desde entonces en una de las características centrales de la forma de concebir los sistemas económicos y administrar las organizaciones. En este sentido, Díaz (1996) caracteriza dos clases de flexibilidad: una "macro" referida al sistema económico y productivo en general que busca adaptarse a las transformaciones del contexto global, y una "micro" o de tipo empresarial que implica el esfuerzo de las organizaciones por adaptarse a las condiciones cambiantes del entorno económi-

$2 \quad$ El término fordismo es usado en la literatura gerencial para referirse al sistema de producción y gestión empresarial predominante en el siglo xx, caracterizado por la producción en serie con énfasis en la especialización en las funciones de los cargos. Toma su nombre de Henry Ford y se fundamenta en los postulados del taylorismo. co. En ésta última, el autor propone tres tipos de flexibilización de las organizaciones: 1) de gestión estratégica, financiera y comercial, 2) de gestión productiva y 3) flexibilidad laboral interna. Esta última, entendida como "la capacidad de la empresa para contratar y utilizar flexiblemente la mano de obra, en sus dimensiones numérica, salarial, funcional y de tipo de contrato" (Díaz, 1996, p. 41).

La flexibilidad laboral se refiere no solo a la capacidad que pueda tener una empresa para contratar o despedir de manera ágil grupos de trabajadores, sino al desarrollo de una filosofía de gestión orientada a lograr capacidades de adaptación y respuestas oportunas a las condiciones permanentemente cambiantes del mercado. La flexibilidad laboral implica formas de administrar el personal que permitan a las organizaciones poder hacer cambios rápidos cuando las circunstancias así lo requieran. En palabras de Sennett (2004), designa la capacidad de las organizaciones para ceder y recuperarse, para adaptarse a las circunstancias cambiantes sin dejar que éstas le afecten de manera drástica que la puedan destruir.

La flexibilidad laboral se presente de múltiples formas en la gestión de personas en las organizaciones. Por ejemplo, los servicios de outsourcing y otras formas atípicas de empleo como el empleo temporal, el trabajo a tiempo parcial, el trabajo temporal a través de agencia de intermediación o la prestación de servicios profesionales; portafolios de condiciones salariales; franjas de horarios de trabajo disponibles para elección de los trabajadores; teletrabajo; trabajo a domicilio; movilidad de los trabajadores a través de diferentes roles derivados de la gestión por proyectos, entre otras.

La individualización en las relaciones de trabajo ha sido una de las consecuencias de la flexibilización laboral, fenómeno que también se circunscribe en el contexto de los procesos de individualización de las sociedades industrializadas mencionado anteriormente. Por lo que las tradicionales relaciones colectivas de trabajo han sido modificadas debido al crecimiento del individualismo y la flexibilidad (Guest, 2004).

Una de las estrategias que tipifica los procesos de individualización en las relaciones de trabajo es el 
modelo de gestión por competencias. Este ha emergido en la literatura gerencial como el paradigma representativo del desempeño de los trabajadores en la actualidad. Tanto así "que al parecer ninguna empresa que se pretenda 'moderna' en la gestión de sus recursos humanos pudiera prescindir" (Soto, 2009 , p. 107). El modelo de gestión por competencias focaliza fuertemente el análisis del trabajo en cada individuo, permitiendo hacer una gestión individualizada del desempeño organizacional y un mayor control en la administración de cada uno de los trabajadores al interior de las organizaciones. En otras palabras, el modelo competencias individualiza la gestión de las personas en las organizaciones.

Otra de las condiciones actuales que caracteriza la individualización en las relaciones de trabajo es el desarrollo de la carrera laboral. La gestión de la carrera laboral ha pasado de estar determinada por el vínculo con las organizaciones y bajo la responsabilidad de éstas, a estar centrada en la relación persona-trabajo (dentro y fuera de las empresas) con mayor responsabilidad por parte de las personas (Ribeiro, 2014). En este sentido, el desarrollo de una carrera laboral deja de ser una responsabilidad de las organizaciones para centrarse en un compromiso de vida por parte de las personas, quienes ahora tienen "la libertad" de gestionar su propia carrera, incluso más allá de los linderos de una sola organización (Louffat, 2005).

En la actualidad toman fuerza los "modelos emergentes de carrera" (Bendassolli, 2009), tales como "carrera sin fronteras" (Arthur, 1994; Arthur \& Rousseau, 1996), "carrera proteica" (Hall, 1996; 2004), "carrera multidireccional" (Baruch, 2004) y "carrera caleidoscópica" (Mainiero \& Gibson, 2017; Mainiero \& Sullivan, 2005). Estas nuevas formas de concebir la carrera laboral son propuestas que buscan dar respuesta a las transformaciones ocurridas desde los años 70 en el trabajo, las organizaciones y las sociedades industriales y que han producido condiciones de incertidumbre, riesgo, fragilización y ruptura de los vínculos tradicionales de trabajo (Bendassolli, 2009).

Estas formas emergentes de carrera son modelos que caracterizan la manera como se establecen las relaciones empresas-trabajadores en un contexto económico en el que la responsabilidad por el propio desarrollo laboral se ha centrado en el individuo. Estas condiciones tienen también efectos de carácter económico para las personas, ya que ellas necesitan mantener vigencia de sus competencias laborales como estrategia de competitividad en los mercados de trabajo, por lo que deben recurrir a sus recursos financieros personales para invertir en la cualificación y actualizaciones necesarias, pues el conocimiento y las capacidades personales requeridas se desactualizan muy rápidamente.

Sumado a la concepción del desarrollo de la carrera laboral centrada en el individuo, se encuentra el concepto de "empleabilidad", el cual ha llegado a configurarse "como una categoría de práctica para dar cuenta de las dinámicas de inclusión-exclusión de los individuos en el mercado de trabajo" (Rentería \& Malvezzi, 2008, pág. 320). Aunque el término sugiere la noción de empleo como forma típica de la vinculación laboral mediada por un contrato de trabajo, realmente este concepto está orientado a las características que debe reunir un individuo para insertarse y mantenerse vigente en el mercado de trabajo, independientemente del tipo de vinculación laboral, ya sea mediante contrataciones directas con las empresas o a través de mecanismos de tercerización laboral.

En este sentido, un individuo es "empleable" o tiene un alto nivel de empleabilidad, si reúne las características que le demanda el mercado de trabajo. Esto es, si tiene las competencias laborales y adecuadas que las organizaciones requieren para que pueda desempeñarse con efectividad, pero a su vez implica la adaptación y transformación permanente de sí mismo como sujeto laboral para mantenerse actualizado en conocimientos y habilidades según vayan cambiando las condiciones de los contextos de trabajo.

Este hecho lleva implícita la idea de que la vinculación, permanencia o desvinculación de un individuo de los mercados de trabajo, depende única y exclusivamente de su propia competencia y no de las condiciones económicas estructurales de un país que posibilita la oferta de puestos de trabajo. Así mismo, el concepto de empleabilidad sugiere también la idea de una estrategia gerencial de 
transferir desde las organizaciones hacia los individuos la responsabilidad por su no contratación o despido (Carrieri \& Sarsur, 2004). En este orden de ideas, "es cada trabajador quien está llamado a desarrollar su propia empleabilidad, en un mercado abierto y móvil, a través de estrategias individuales de desarrollo del propio 'capital' de competencias" (Soto, 2009, p. 108).

En este sentido, como lo afirman Beck y BeckGernsheim (2003), "en la actualidad abundan las directrices -en el sistema educativo, el mercado de trabajo o el Estado del bienestar- que contienen la exigencia de que el individuo tome las riendas de su propia vida so pena de sanción económica" (p. 72); esta es una de las características más representativas de la individualización en las relaciones de trabajo.

En síntesis, como bien lo afirma Bauman (2003), "la 'individualización' consiste en hacer que la 'identidad' humana deje de ser un 'dato' para convertirse en una 'tarea', y en cargar sobre los actores la responsabilidad de la tarea y las consecuencias (y efectos secundarios) de su actuación (p. 20).

\section{Implicaciones para la gestión de recursos humanos}

Se entiende la gestión de recursos humanos como un enfoque estratégico, integrado y coherente que orienta la vinculación, el desarrollo y el bienestar de las personas que trabajan en las organizaciones, con el propósito de llevar a cabo la estrategia de negocios y alcanzar los objetivos corporativos (Armstrong, 2014).

Tanto en la literatura sobre gestión empresarial como en la dedicada a la administración estratégica del personal, se ha puesto en evidencia la importancia de las personas para los resultados de las organizaciones "y la conexión incuestionable del desempeño laboral con la obtención de mejores resultados empresariales" (Morales-Fernández, Ariza-Montes, \& Morales-Gutiérrez, 2013, p. 310). Sin embargo, en contextos de individualización, la gestión de recursos humanos debe orientarse a dar un mayor énfasis a la gestión individualizada de las relaciones de trabajo.
Hace ya dos décadas Pfeffer (2000) se preguntaba:

¿Qué importancia tienen los textos sobre la cultura de la organización en un mundo en el que una elevada proporción de la fuerza laboral trabaja medio tiempo o como contratistas independientes y tiene un apego tan limitado a las compañías que la cultura y el control cultural están prácticamente fuera de lugar? (p. 22).

Ampliando esta reflexión a otros ámbitos de la gestión de recursos humanos, es posible ver cómo las formas flexibles de administración del personal, los mecanismos de tercerización de la fuerza laboral, el debilitamiento de los colectivos sindicales, la emergencia del concepto de empleabilidad, el paradigma de la gestión por competencias y el mayor énfasis de los trabajadores en la gestión de su carrera laboral, en medio de un contexto social en el que ocurre una fragilización de los vínculos humanos, las estrategias tradicionales de gestión de recursos humanos basadas en colectivos sociales pierden vigencia para un contexto que ha cambiado.

La fragilización de las relaciones laborales es un hecho evidente en las sociedades industrializadas. Esta fragilización se traduce en una individualización de las relaciones de trabajo, es decir, "un individuo que se convierte en el único responsable de su vida laboral, un individuo que negocia directamente sus condiciones con la empresa ya que lo que se busca es la negociación directa e individual entre jefe y empleado" (Tovar \& Revilla, 2012, p. 236).

En este orden de ideas, la gestión de recursos humanos en la actualidad debe orientarse más a la experiencia y vivencia de trabajo visto de manera individual, en lugar de preferir acciones genéricas dirigidas de manera colectiva. No se trata de hacer una administración centrada exclusivamente en el individuo, puesto que la gestión de equipos es una estrategia importante en las organizaciones contemporáneas. El asunto clave que debe ser tenido en cuenta es que en la actualidad las estructuras de recursos humanos son altamente flexibles, con un importante componente de la fuerza laboral integrada mediante formas de tercerización, con sistemas de gestión por competencias que centran en el individuo, la incorporación, la evaluación y el control de resultados, además de una mayor res- 
ponsabilización individual por el desarrollo de la carrera laboral.

En el ámbito de la gestión de recursos humanos "la experiencia del empleado" en el contexto de trabajo es clave para su compromiso y participación (Deloitte, 2017). Como bien lo indica esta organización "al día de hoy, las organizaciones están mirando la travesía del colaborador, estudiando las necesidades de su fuerza laboral, y usando indicadores (net promoter scores-NPS) para entender su experiencia dentro de la organización" (Deloitte, 2017, p. 7). Así, "enfocándose en la experiencia del empleado, los líderes de RH pueden mejorar el compromiso, empoderar a equipos y líderes, y desarrollar soluciones de fuerza laboral que podrán ser útiles e irresistibles para los empleados" (Deloitte, 2017, p. 59). Aquí se presentan tres alternativas que orientan el desarrollo de estrategias de gestión centradas en el individuo.

La primera se refiere a la perspectiva de los "contratos idiosincrásicos", que implican acuerdos individualizados respecto de aspectos en la relación de trabajo como compensaciones, horarios, oportunidades de capacitación y desarrollo, entre otros. Los cuales van dirigidos solo a algunos de los trabajadores de la organización considerados como elementos clave debido a sus competencias o rol estratégico. "Los contratos idiosincrásicos son un medio para reconocer, recompensar y motivar a aquellos empleados que agregan un valor diferencial a la organización, pero no constituyen una práctica para otorgar un trato preferencial en función de vínculos políticos o personales" (Dabos \& Rivero, 2012, p. 5).

En este orden de ideas, aunque las organizaciones definen políticas de recursos humanos que orientan las relaciones de trabajo y establecen criterios para procesos de selección, compensación, evaluación y bienestar, entre otros, incluso en el marco de las regulaciones jurídicas gubernamentales, también es factible la realización de acuerdos específicos individuales que sean estratégicos para las organizaciones.

Este tipo de contratos se definen como "los acuerdos voluntarios personalizados de negociaciones de naturaleza no estándar entre los empleados y sus empleadores respecto de los términos que beneficien a cada parte" (Rousseau, Ho, \& Greenberg, 2006, p. 978). Pueden ser negociados desde instancias directivas de la organización como la Gerencia General o la Dirección de Recursos Humanos, o pueden constituirse en acuerdos particulares entre un trabajador y su jefe inmediato. Sin embargo, las investigaciones sugieren que los términos idiosincrásicos en los contratos de trabajo son negociados generalmente por directivos de niveles gerenciales medio y alto (Dabos \& Rivero, 2012). Así mismo, los acuerdos idiosincrásicos pueden darse al comienzo de la relación laboral o negociarse en algún momento específico cuando el individuo ya forma parte de la organización; en este caso, es posible que el acuerdo se constituya en algún punto de cambio importante en el ejercicio de los roles laborales que ejecuta el trabajador en la organización.

Rosen, Slater y Chang (2013) proponen cuatro dimensiones para tener en cuenta en el momento de evaluar la posibilidad de realizar acuerdos idiosincrásicos con los trabajadores, esto son: flexibilidad en los horarios, flexibilidad en la ubicación, incentivos financieros $y$, tareas y responsabilidades laborales, aspectos relacionados respectivamente con el cuándo, dónde, por qué y qué hacen los empleados en su trabajo. Por su parte, Dabos y Rivero (2012) examinaron la presencia de términos idiosincrásicos en los contratos de trabajo celebrados por trabajadores en organizaciones intensivas del conocimiento en Argentina, y encontraron que los aspectos más negociados en sus contratos eran la oportunidad del desarrollo de competencias laborales específicas y flexibilidad en la jornada laboral. Por el contrario, los aspectos menos negociados fueron la posibilidad de trabajar solo en aquellas áreas acordes con los intereses profesionales. Cabe resaltar en este estudio un importante número de casos presentados respecto de salarios diferenciales por competencias altamente demandadas (31 casos), la flexibilidad en la jornada laboral ( 25 casos) y flexibilidad de tiempos en relación con los períodos vacacionales (15 casos), de 184 personas que respondieron la encuesta.

Los contratos idiosincrásicos, en síntesis, facilitan la construcción de relaciones individualizadas entre los gerentes y altos directivos de las organizacio- 
nes con los trabajadores de estas. Igualmente, "un tratamiento más idiosincrásico de los empleados basado en las necesidades personales puede generar relaciones de calidad con los supervisores y las organizaciones" (Singh \& Vidyarthi, 2018, p. 10). En este sentido, la investigación empírica ha evidenciado que este tipo de acuerdos influyen en las percepciones, actitudes y comportamientos relacionados con el desempeño de los trabajadores, tales como la satisfacción, el compromiso afectivo, la motivación y la confianza y credibilidad en la organización (Liao, Wayne, \& Rousseau, 2014).

Una segunda alternativa se relaciona con el ejercicio del liderazgo. Tradicionalmente este ha sido visto en una relación individuo-grupo, en el sentido de que diversos autores lo han definido en términos del grado de influencia de un individuo sobre un grupo determinado. En el contexto actual de individualización de las relaciones de trabajo, el liderazgo individualizado emerge como una importante perspectiva que responde a las características de las sociedades laborales actuales. Esta perspectiva se enfoca en las relaciones diádicas que ocurren entre un superior y un subordinado específico y que derivan en un conjunto de intercambios entre ellos, siendo clave la confianza entre las partes y el apoyo brindado por el líder (Yammarino \& Dansereau, 2002).

El liderazgo individualizado se basa en la teoría del intercambio líder-miembro (LMX por sus siglas en inglés) (Dansereau Jr, Graen, \& Haga, 1975). Esta teoría se apoya en el supuesto de que los líderes influyen en los empleados a través de la calidad de las relaciones que desarrollan con ellos; más específicamente, se ha encontrado que los líderes desarrollan relaciones de calidad variable con sus trabajadores, lo que implica que existen características diferenciales en las relaciones que establecen los líderes con los diferentes miembros de un grupo determinado (Erdogan \& Bauer, 2015).

Algunos de los aspectos clave en las relaciones diádicas para un ejercicio eficaz del liderazgo individualizado son el apoyo y comprensión por parte del jefe según lo perciben los empleados, la calidad en la relación interpersonal incluida la comunicación y el grado de cercanía personal entre líder y seguidor
(Wallis, Yammarino, \& Feyerherm, 2011). En este orden de ideas, dado que el liderazgo individualizado se fundamenta en la relación diferenciada de un líder con los diversos miembros de su equipo de trabajo, la calidad de la interacción se convierte en un rasgo fundamental en el ejercicio del liderazgo.

Finalmente, como estrategia para la gestión de recursos humanos en contextos de individualización de las relaciones de trabajo, se encuentra la gestión a través de los contratos psicológicos. Como lo sugiere Malvezzi (2011), una de las características clave de la sociedad del siglo xxı es "la sustitución de la dependencia de instituciones colectivas, como las tradiciones e ideologías, por la negociaciones y contratos psicológicos" (p. 68). Para este autor, la profundización en las condiciones de individualización que caracterizan las relaciones humanas en la actualidad produce un efecto en la fragilización del vínculo de las personas con las instituciones. Así, se requiere de otros mecanismos reguladores de las relaciones interpersonales, y "la construcción de vínculos a través de los contratos psicológicos gana esa función" (Malvezzi, 2011, p. 68).

Aunque el concepto de contrato psicológico aparece en la literatura de las Ciencias Sociales en la década de los 60 por parte de Argyris (1960) y entre los años 70 y 90 su uso en la literatura especializada pierde vigencia, en las últimas tres décadas ha tenido una importante prominencia en las publicaciones en el campo de la psicología de las organizaciones, ya que este es "es un concepto clave en la comprensión de las relaciones de trabajo en la actualidad" (Rentería \& Vesga, 2019, p. 129), puesto que este "es considerado como una vía útil de comprensión y quizá de administración de los cambios evidentes de las relaciones de trabajo causados por las nuevas circunstancias económicas y organizacionales" (Conway \& Briner, 2009, p. 7); esto es posible debido a que este concepto se centra en la comprensión de las relaciones de los trabajadores con las organizaciones y actúa como un mecanismo de regulación y reconstrucción de los vínculos laborales (Malvezzi, 2011).

El contrato psicológico se refiere al "conjunto de compromisos implícitos en la relación de trabajo, más allá de los acuerdos formales explícitos 
establecidos entre las partes en el marco de un acuerdo de relación laboral” (Rentería \& Vesga, 2019 , p. 130), lo cual supone un conjunto de deberes y expectativas que regulan e inciden en la calidad de las relaciones establecidas entre los trabajadores y las organizaciones a las que están vinculados.

Como estrategia de gestión, "el contrato psicológico se encuentra imbricado en la dinámica de las organizaciones ocupadas en estrechar las relaciones del individuo con el ambiente de trabajo, considerado el elemento que vincula empleado-organización" (Pitombo \& Albuquerque, 2011, p. 20). En tal sentido, la gestión de recursos humanos a través de los contratos psicológicos se constituye en una importante herramienta de administración estratégica, puesto que se ha identificado que "la percepción de ruptura del contrato tiene consecuencias negativas y muy consistentes, tanto en las actitudes como en las conductas de los empleados" (Topa \& Palací, 2004, p. 168), ya que según estos autores, ante la percepción de ruptura hay una disminución de las conductas de ciudadanía organizacional y menor compromiso con la organización.

La importancia de la gestión del contrato psicológico como estrategia de la gerencia de los recursos humanos, se fundamenta en la necesidad de las organizaciones de lograr compromiso por parte de sus trabajadores en un contexto de alta competitividad en el que predominan condiciones de flexibilización laboral y acelerados cambios, ya que la gestión de recursos humanos a través del contrato psicológico se sustenta en el compromiso de bienestar psicológico, personal y profesional entre los trabajadores y la organización (Pallarès \& Selva, 2016).

\section{A modo de conclusión}

La evolución de las sociedades industrializadas ha producido transformaciones en las formas de relacionamiento y en la calidad de los vínculos humanos, produciendo una tendencia hacia la fragilización de estos y la individualización en las relaciones. Una de las características de las sociedades actuales es la consolidación del paso de una "solidaridad mecánica" centrada en identidades colectivas, a una "solidaridad orgánica" focalizada en la complementariedad de los individuos. En tal sentido, puede afirmarse que las conciencias colectivas se han debilitado y hoy emergen con fuerza procesos de individualización.

En contextos de trabajo, cada trabajador en una organización es un individuo único, con su propia historia, anhelos y expectativas, que configura de manera personal contratos psicológicos con las organizaciones a las cuales se vincula. Si los dirigentes organizacionales quieren lograr compromiso y lealtad por parte de los trabajadores, deben permitirles realizarse como individuos a través de su trabajo; la vida en la organización debe ser parte de su proyecto de vida, aunque sea temporal, o convertirse en una fase de su proceso evolutivo y de su desarrollo como ser humano.

Es entonces labor de los dirigentes, elaborar mecanismos de articulación de las individualidades para complementar sus competencias. Así como en un concierto musical cada instrumento emite un sonido particular y único y opera de una manera diferente a todos los demás, pero que por medio de la habilidad dirigente de su director todos suenan en armonía produciendo la magia de la música manifestando melodía, armonía y ritmo.

Un colectivo humano no es una unidad compacta en la que los elementos que lo conforman piensan igual y actúan al unísono como autómatas que responden a una programación. Un colectivo tampoco es una agrupación de individuos desconectados, encerrado cada uno en su propio mundo sin conciencia del entorno que le rodea y actuando cada uno por su lado. Un colectivo efectivo es un conjunto de individuos que desarrolla cada uno su máximo potencial, pero que están sincronizados para complementarse mutuamente como producto del actuar del dirigente. El concepto clave es conexión, aunque siendo diferentes y actuando con conciencia individual, cada individuo está conectado con los otros.

Una gestión de recursos humanos con una perspectiva idiosincrásica, más centrada en los individuos, sus necesidades particulares y contratos psicológicos individuales, permitirá desarrollar al máximo su potencial para articularlo con los potenciales de desempeño del resto de individuos en la organización, para lograr una mayor efectividad en el alcance de los objetivos estratégicos. 


\section{Referencias}

Argyris, C. (1960). Understanding Organizational Behavior. Homewood: Dorsey.

Armstrong, M. (2014). Armstrong's handbook of human resource management practice (13th ed.). London: Kogan Page.

Arthur, M. (1994). The boundaryless career: a new perspective for organizational inquiry. Journal of Organizational Behavior, 15, 295306. https://doi.org/10.1002/job.4030150402

Arthur, M. \& Rousseau, D. (1996). The Boundaryless Career: A new employment principle for a new organizational era. New York: Oxford.

Baruch, Y. (2004). Transforming careers: from linear to multidirectional career paths. Career Development International, 9(1), 58-73. https:// doi.org/10.1108/13620430410518147

Bauman, Z. (2003). Individualmente, pero juntos. En U. Beck, \& E. Beck-Gernsheim, La individualización. El individualismo institucionalizado y sus consecuencias sociales y políticas (pp. 1926). Barcelona: Paidós.

Bauman, Z. (2007). Amor líquido: Acerca de la fragilidad de los vínculos humanos. México, D.F.: Fondo de Cultura Económica.

Beck, U. (1997). La reinvención de la política: Hacia una nueva teoría de la modernidad relfexiva. In U. Beck, A. Giddens, \& S. Lash, Modernización reflexiva. Política, tradición y estética en el orden social moderno (pp. 13-73). Madrid: Alianza Editorial.

Beck, U. (1998). La sociedad del riesgo. Hacia una nueva modernidad. Barcelona: Paidós.

Beck, U. \& Beck-Gernsheim, E. (2003). La individualización. El individualismo institucionalizado y sus consecuencias sociales y políticas. Barcelona: Paidós.

Bendassolli, P. F. (2009). Recomposição da relação sujeito-trabalho nos modelos emergentes de carreira. Revista de Administração de Empresas, 49(4), 387-400.
Boltanski, L. \& Chiapello, È. (2002). El nuevo espíritu del capitalismo. Madrid: Akal.

Carrieri, A. \& Sarsur, A. (2004). Percurso Semântico do Tema Empregabilidade: a(Re)Construção de Parte da História de uma Empresade Telefonia. Revista de Administração Contemporânea, 8(1), 129-150. https://doi.org/10.1590/ S1415-65552004000100007

Castells, M. (1999). La Era de la Información: Economía, Sociedad y Cultura. Vol. 1. La sociedad red. México, D. F.: Siglo xxı.

Conway, N. \& Briner, R. (2009). Understanding Psychological Contracts at Work: A critical evaluation of theory and research. New York: Oxford.

Dabos, G. E. \& Rivero, G. A. (2012). Contratos idiosincrásicos en la atracción y retención del talento: tres estudios en organizaciones intensivas en conocimiento de la Argentina. Estudios Gerenciales, 28, 3-12.

Dansereau Jr, F., Graen, G., \& Haga, W. J. (1975). A vertical dyad linkage approach to leadership within formal organizations: A longitudinal investigation of the role making process. Organizational Behavior and Human Performance, 13(1), 46-78. https://doi. org/10.1016/0030-5073(75)90005-7

Deloitte. (2017). 2017 Deloitte Global Human Capital Trends. Reescribiendo las reglas para la era digital. Recuperado de: https://www2.deloitte.com/gt/es/pages/human-capital/articles/ introduction-human-capital-trends-2017.html

Díaz, Á. (1996). Flexibilidad productiva en las economías de la región y nuevos modelos de empresa. Boletín técnico interamericano de formación profesional. oІт/Cinterfor(137), 31-60. Recuperado de: http://www.ilo.org/ public/spanish/region/ampro/cinterfor/publ/ boletin/137/pdf/bol137b.pdf

Durkheim, É. (2012). La división del trabajo social. Madrid: Minerva Ediciones.

Erdogan, B. \& Bauer, T. N. (2015). LeaderMember Exchange Theory. In J. Wright (Ed.), 
International Encyclopedia of the Social \& Behavioral Sciences (2nd ed., Vol. 13, pp. 641647). Waltham: Elsevier.

Gómez, M. A. (2014). Sobre la flexibilidad laboral en Colombia y la precarización del empleo. Diversitas: Perspectivas en Psicología, 10(1), 103-116.

Guest, D. (2004). The Psychology of the Employment Relationship: An analysis based on the Psychological Contract. Applied Psychology, 53(4), 541-555.

Hall, D. (1996). Protean Careers of the 21st Century. The Academy of Management Executive, 10(4), 8-16.

Hall, D. (2004). The protean career: A quartercentury journey. Journal of Vocational Behavior, 65(1), 1-13. https://doi.org/10.1016/j. jvb.2003.10.006

Harvey, D. (1998). La condición de la posmodernidad: Investigación sobre los orígenes del cambio cultural. Buenos Aires: Amorrortu.

Jung, C. G. (1946). The Integration of the Personality. London: Lowe and Brydone Printers.

Liao, C., Wayne, S. J., \& Rousseau, D. M. (2014). Idiosyncratic deals in contemporary organizations: A qualitative and meta-analytical review. Journal of Organizational Behavior, 9-29. https://doi.org/10.1002/job.1959

Louffat, E. (2005). Administrando nuestras propias competencias en un contexto de carreras sin fornteras ni límites. Revista EAN, (54), 85-97.

Mainiero, L. \& Gibson, D. (2017). The Kaleidoscope Career Model Revisited: How Midcareer Men and Women Diverge on Authenticity, Balance, and Challenge. Journal of Career Development, 20(10), 1-17. https://doi. org $/ 10.1177 / 0894845317698223$

Mainiero, L. \& Sullivan, S. (2005). Kaleidoscope careers: An alternate explanation for the "optout" revolution. Academy of Management Executive, 19(1), 106-123.
Malvezzi, S. (2011). A Gestão dos Contratos Psicológicos. Revista do Instituto de Marketing Industrial, 53, 66-73.

Morales-Fernández, E. J., Ariza-Montes, J. A., \& Morales-Gutiérrez, A. C. (2013). La evolución de la gestión de recursos humanos desde una perspectiva estratégica. Revista de Fomento Social, 68, 309-329.

Pallarès, S. \& Selva, C. (2016). La gestión del contrato psicológico en una empresa multinacional de alimentos española. Psicoperspectivas, 15(2), 40-52. https://doi.org/10.XXXX/ PSICOPERSPECTIVAS -VOL15 - ISSUE 2FULLTEXT-685

Pfeffer, J. (2000). Nuevos rumbos en la teoría de la organización. Problemas y posibilidades. México, D.F.: Oxford.

Pitombo, N. R. \& Albuquerque, L. G. (2011). Gestão estratégica de pessoas, comprometimento e contrato psicológico: o caso Vale. Revista de Administração, 46(1), 19-31. https://doi. org/10.5700/rausp0995

Rentería, E. (2012). Desarrollo de la empleabilidad como estrategia para las organizaciones: limitaciones, posibilidades e implicaciones para las personas. Aristeo, 2, 69-90.

Rentería, E. \& Malvezzi, S. (2008). Empleabilidad, cambios y exigencias psicosociales en el trabajo. Universitas Psycologica, 7(2), 319-334.

Rentería, E. \& Vesga, J. J. (2019). Los niveles de actuación en las relaciones de trabajo y la formación de contratos psicológicos. Diversitas: perspectivas en psicología, 15(1), 129-142. https://doi. org/10.15332/s1794-9998.2019.0001.10

Ribeiro, M. A. (2014). Problemas contemporâneos para a psicologia do trabalho e das organizações. En J. Orejuela (Ed.), Psicología de las organizaciones y del trabajo: apuestas de investigación (pp. 47-78). Cali: Bonaventuriana.

Ribes, A. J. (2012). Injusticia, simpatía y ausencia de solidaridad orgánica: una introducción a la 
división del trabajo social de Émile Durkheim. En É. Durkheim, La división del trabajo social (pp. 9-50). Madrid: Minerva Ediciones.

Rodríguez, M., Erbes, A., Roitter, S., \& Pujol, A. (2015). El nuevo trabajo industrial: una mirada crítica desde la calidad del empleo. Sociedad y economía, 28, 137-162.

Rosen, C. C., Slater, D. J., \& Chang, C.-H. (. (2013). Let's Make a Deal: Development and Validation of the Ex Post I-Deals Scale. Journal of Management, 39(3), 709-742. https://doi. org/10.1177/0149206310394865

Rousseau, D., Ho, V., \& Greenberg, J. (2006). I-Deals: Idiosyncratic terms in Employment Relationships. Academy of Management Review, 31(4), 977-994.

Sánchez, J. F. (2008). Los vínculos sociales como formas de regulación. Reflexiones sobre el poder de los vínculos en la sociedad colombiana. Nóesis. Revista de Ciencias Sociales y Humanidades, 17(34), 204-234.

Sennett, R. (2004). La corrosión del carácter. Las consecuencias personales del trabajo en el nuevo capitalismo (7a ed.). Barcelona: Anagrama.

Singh, S. \& Vidyarthi, P. R. (2018). Idiosyncratic Deals to Employee Outcomes: Mediating Role of Social Exchange Relationships. Journal of Leadership \& Organizational Studies, 25(4), 1-13. https://doi.org/10.1177/1548051818762338
Soto, Á. (2009). Formas y tensiones de los procesos de individualización en el mundo del trabajo. Psicoperspectivas, 8(2), 102-119.

Topa, G. \& Palací, F. J. (2004). ¿Ruptura o cumplimiento del contrato psicológico? Una revisión meta-analítica de la investigación empírica. Acción Psicológica, 3(3), 155-171.

Tovar, F. J. \& Revilla, J. C. (2012). La institucionalización de la individualización del trabajo: el concepto de flexiguridad. Cuadernos de Relaciones Laborales, 30(1), 235-258. https:// doi.org/10.5209/rev_CRLA.2012.v30.n1.39122

Vesga, J. J. (2019). Identidad colectiva: un concepto lábil en el contexto de las relaciones de trabajo en la actualidad. En M. García (Ed.). Actualizaciones en psicología organizacional (pp. 11-22). Bogotá, D.C.: Editorial Universidad Católica de Colombia.

Wallis, N. C., Yammarino, F. J., \& Feyerherm, A. (2011). Individualized leadership: A qualitative study of senior executive leaders. The Leadership Quarterly, 22(1), 182-206. https:// doi.org/10.1016/j.leaqua.2010.12.015

Yammarino, F. \& Dansereau, F. (2002). Individualized Leadership. Journal of Leadership \& Organizational Studies, 9(1), 90-99.

Zabludovsky, G. (2013). El concepto de individualización en la sociología clásica y contemporánea. Política y Cultura, 39, 229-248. 